\title{
Using a QR Code to Register Voters in a Family Medicine Residency Clinic
}

Yumiko Nakamura, MD, MPH | Vishnu Muppala, MD, MPH | Bich-May Nguyen, MD, MPH

PRiMER. 2021;5:27.

Published: 8/23/2021 | DOI: 10.22454/PRiMER.2021.519685

\section{To the Editor:}

Civic engagement is increasingly recognized as an important social determinant of health. Multiple studies have found associations between health status, including self-reported health or disability, and voting habits. ${ }^{1,2}$ The disparity of voting among less healthy populations, many of whom are socially and economically disenfranchised, leads to skewed electoral outcomes and eventually, health policy that impacts these communities. ${ }^{1}$ Health care spaces provide an opportunity to promote civic participation among historically underrepresented patient populations. ${ }^{1}$ Because family medicine serves a broad patient population in a variety of settings, family physicians are uniquely positioned to provide nonpartisan opportunities for civic engagement and voting in a trusted community space. Over the last decade, voter registration initiatives in clinics have been growing and a handful have been documented in the medical literature. ${ }^{3}$

Because of this, we attempted an intervention to promote civic engagement in a family medicine residency clinic in a suburb of Houston. Flyers in English and Spanish were posted on bulletin boards in over 50 clinic exam rooms in a patient-centered medical home. The flyers displayed customized QR codes that redirected users to the vote.org website, where participants could verify their voter registration status and complete an online request for a voter registration form or absentee ballot to be mailed to their home. The goal of the pilot program was two-fold. The first objective was to promote voter registration and civic empowerment among patients and health care workers in an accessible health care setting. The second objective was to assess QR codes as a viable and convenient medium for voter registration in clinics.

Over the span of 2 months, our QR codes had only 20 interactions. Still, a high proportion of participants who interacted with the QR codes completed the online form. We were unable to determine if the participants mailed the paper form to complete the registration or absentee ballot request, nor do we know if they voted. While it seems feasible to obtain stakeholder investment and implement voter registration in family medicine clinics, more active programming may be required in the future. This is consistent with other efforts finding impersonal methods to be ineffective in mobilizing voters and dynamic interaction of authentic person-toperson contact being the most important component in successful outreach. ${ }^{4}$ Therefore, posting QR codes alone may not promote engagement, whether it be voter registration or enrollment in other services in clinic settings.

Despite the underwhelming response, the initiative was a meaningful attempt at exploring further opportunities for family medicine practitioners to promote patient and community empowerment. With the physician platform, it is imperative that we accept the full scope of responsibility in protecting the health of our patients and community. Facilitating patient access to engage with the systems that determine the policies that both 
directly and indirectly affect their health and well-being is undeniably a part of our duty as family physicians.

\section{Author Affiliations}

Yumiko Nakamura, MD, MPH - Family and Community Medicine Residency Program, University of California San Francisco

Vishnu Muppala, MD, MPH - Emergency Medicine Residency Program, Maimonides Medical Center, Brooklyn, NY

Bich-May Nguyen, MD, MPH - University of Houston College of Medicine, Houston, TX

\section{References}

1. Brown CL, Raza D, Pinto AD. Voting, health and interventions in healthcare settings: a scoping review. Public Health Rev. 2020;41(1):16. doi:10.1186/s40985-020-00133-6

2. Blakely TA, Kennedy BP, Kawachi I. Socioeconomic inequality in voting participation and self-rated health. Am J Public Health. 2001;91(1):99-104. doi:10.2105/AJPH.91.1.99

3. Liggett A, Sharma M, Nakamura Y, Villar R, Selwyn P. Results of a voter registration project at 2 family medicine residency clinics in the Bronx, New York. Ann Fam Med. 2014;12(5):466-469. doi:10.1370/afm.1686

4. Lessons from GOTV experiments. Yale University Institute for Social and Policy Studies. Accessed July 7, 2021. https://isps.yale.edu/node/16698

Copyright $@ 2021$ by the Society of Teachers of Family Medicine 